\title{
Power Control Algorithm Based on a Cooperative Game in User-Centric Unmanned Aerial Vehicle Group
}

\author{
Yuexia Zhang $\mathbb{D}^{1,2,3}$ and Pengfei Zhang $\mathbb{D}^{1}$ \\ ${ }^{1}$ School of Information and Communication Engineering, Beijing Information Science \& Technology University, \\ Beijing 100101, China \\ ${ }^{2}$ Key Laboratory of Modern Measurement \& Control Technology, Ministry of Education, \\ Beijing Information Science \& Technology University, Beijing 100101, China \\ ${ }^{3}$ Beijing Key Laboratory of High Dynamic Navigation Technology, University of Beijing Information Science \& Technology, \\ Beijing, 100101, China \\ Correspondence should be addressed to Yuexia Zhang; zhangyuexia@bistu.edu.cn
}

Received 8 April 2021; Accepted 11 May 2021; Published 21 May 2021

Academic Editor: Fei Xiong

Copyright (c) 2021 Yuexia Zhang and Pengfei Zhang. This is an open access article distributed under the Creative Commons Attribution License, which permits unrestricted use, distribution, and reproduction in any medium, provided the original work is properly cited.

\begin{abstract}
The quality of service (QoS) of a user in user-centric unmanned aerial vehicle group (UUAVG) is degraded by complex cochannel interference; hence, a cooperative game power control (CGPC) algorithm in UUAVG is proposed. The algorithm helps to establish a downlink power control model of the UUAVG, construct a product of the signal to interference noise ratio function of each user as a utility function of the cooperative game, and deduce the optimal power control scheme using the Lagrange function. This scheme reduces the interference of the service unmanned aerial vehicle (UAV) to edge users and improves the communication quality of all the users as well as the throughput of the entire system. Simulation results show that the average throughput of the CGPC algorithm improved by $10.32 \%$ compared with the traditional Stackelberg Game based Nonunified Pricing Power Control (SGNPPC) algorithm. This shows that the CGPC algorithm can effectively reduce the transmission power of the cooperative UAV and enhance the capacity of the entire system, ensuring communication quality.
\end{abstract}

\section{Introduction}

With the rapidly increasing number of wireless devices and growth of user traffic demand, improving the throughput of a communication system has gained considerable attention [1]. Wireless networks are effective solutions to improve system throughput because a large number of unmanned aerial vehicles (UAVs) groups can be deployed. The usercentric UAV group (UUAVG) can not only improve system capacity but also achieve seamless coverage and enhance the quality of service (QoS) of the system [2]. The UUAVG network organises a dynamic UAVG for each user; the unmanned aerial vehicles group (UAVG) is composed of all potential UAVs near a user, so that the user can perceive that the network follows them [3]. UUAVG is defined as the network architecture of serving user by the "decellular" method [4]. When the user moves, the UAVG will dynamically add and delete UAV members according to the user location to meet user requirements in the UAVG network centre [5]. Compared with traditional cellular network, UUAVG network structure can effectively improve network coverage, reduce the interrupt probability by $30 \%$, and improve the spectrum efficiency by $5 \%-15 \%[6,7]$. Although the UUAVG improves the system throughput by deploying a large number of UAVs, the cochannel interference problem is severe and reduces the QoS for the users. Therefore, it is essential to realise methods to reduce this cochannel interference.

At present, many scholars have studied the complex interference problem of UUAVG, and power control is regarded as the most effective interference suppression method. In [8], an orthogonal frequency division 
multiplexing- (OFDM-) based UAVG communication link resource allocation algorithm was introduced. This algorithm considers subcarrier and power allocations. It maximises the system capacity, while accounting for user fairness. However, the algorithm is complicated and has a low convergence speed. In [9], the local optimal solution is obtained by joint optimisation of uplink cell association and power allocation, which effectively improves the network performance. In [10], aiming at the problem of UAV access and base station bandwidth allocation, a hierarchical game power control algorithm is proposed. This algorithm can effectively solve the problem of UAV access and base station bandwidth allocation. Nash equilibrium is proved by theory, but the problem is modeled as a noncooperative game, sacrificing the overall performance of the system. In [11], a power control algorithm based on mean field game and deep reinforcement learning is proposed. The algorithm first transforms the power control problem into discrete mean constant game and then uses neural network to solve the optimal transmit power. Although this algorithm can effectively improve the energy efficiency of UAV, its complexity is much higher than those of other algorithms. In [12], a noncooperative game distributed power control algorithm was introduced, which could effectively improve the system throughput and reduce cochannel interference; however, the proposed algorithm cannot achieve optimal system capacity. In [13], a power control algorithm based on noncooperative game is proposed. The algorithm adopts a nonuniform pricing method and sets different prices for different base stations. The scheme can effectively improve the system performance in UAVG network structure. Although the above studies reduce interference to a certain extent, they are not user-centric and cannot achieve optimal system throughput.

To solve the above problems, a cooperative game power control algorithm in UUAVG is proposed by this study. First, the system model of the UUAVG downlink power control is established. Considering the interference to user QoS and service UAV to edge users, a new cooperative game utility function is proposed, and the optimal power control scheme is solved using the Lagrange function. The simulation results show that the cooperative game power control (CGPC) algorithm can reduce the user transmitted power and interuser interference and improve system throughput.

\section{System Model}

The model of UUAVG downlink power control system is shown in Figure 1. Assuming that there is a service user (SU) in the model, the dynamic UAVG is formed with the SU as the centre, and the coverage range of the UAVG available to the $\mathrm{SU}$ is represented by the dotted circle. It is assumed that there is only one service unmanned aerial vehicle (SUAV) in the UAVG. Suppose that there are $n$ cooperative unmanned aerial vehicles (CUAV), denoted as CUAVi $(i=1,2, \ldots$, $i, \ldots, j, \ldots, N)$; in the coverage of the UAVG, assume that there are $n$ edge users (EU) at the same time, denoted as EUi

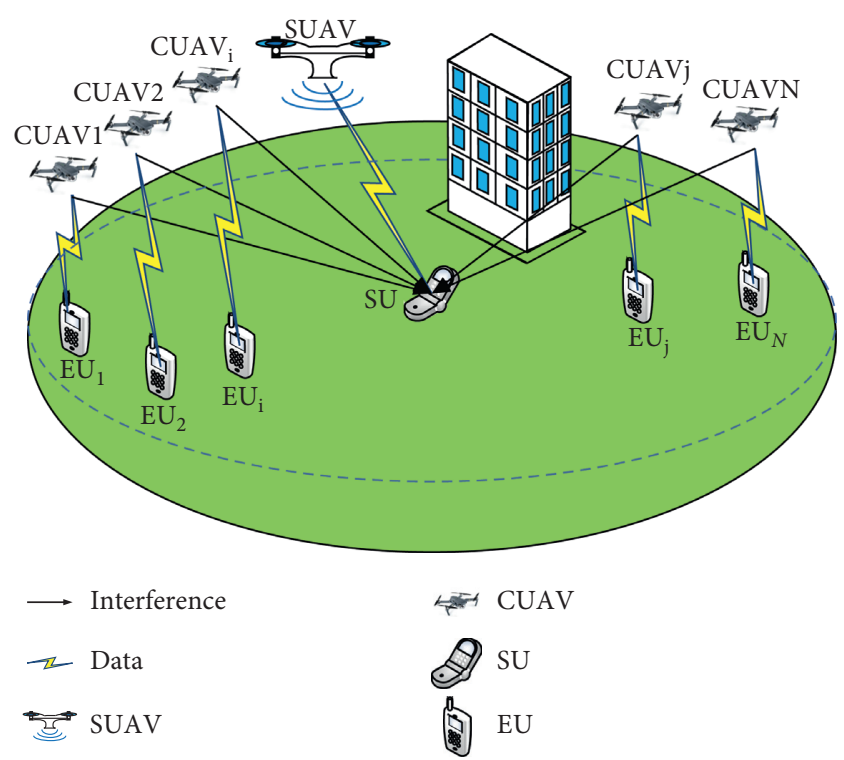

Figure 1: Downlink power control system model of UUAVG.

$(i=1,2, \ldots, i, \ldots, j, \ldots, N)$, and that they are far away from the SUAV, with the service base station of EUi represented as CUAVi. Let the vertical height of the SUAV and CUAV from the ground be $H_{0}$. Owing to the scarcity of spectrum availability and to improve frequency utilisation, when deploying the UAVG, the downlink communication frequencies of the SUAV and SU are the same as those of the CUAVi and EUi. Therefore, the interference between the SUAV and CUAV and those between the CUAVs are inevitable.

For each user, they are in the center of the network, and UAVs around the user are dynamically composed of UAVG. Each user has a unique UAVG to provide services, but each UAV may belong to a different UAVG at the same time. Therefore, each UAV may be both a user's SUAV and another user's CUAV at the same time. For users, if a UAVG is the user's own UAVG, it is called a service user of this UAVG; otherwise, it is called an edge user of this UAVG.

Let $p_{t}$ and $p_{i}$ denote the transmitted power levels of SUAV and CUAVi, and let $\gamma_{i}$ denote the signal to interference noise ratio (SINR) of the corresponding EUi.

$$
\gamma_{i}\left(p_{i}\right)=\frac{p_{i} h_{i i}}{\sum_{j=1, j \neq i}^{N} p_{j} h_{j i}+p_{t} g_{s i}+\sigma^{2}} .
$$

The downlink channel gain between CUAVi and EUi is denoted as $h_{i i}$, the channel gain between CUAV and EUi is denoted as $h_{j i}$, and the channel gain between SUAV and EUi is denoted as $g_{s i}$. The power of the additive white Gaussian noise on EUi is $\sigma^{2}$. The distance between CUAVi and EUi is denoted as $d_{i i}$ and that between SUAV and EUi is denoted as $r_{s i}$.

The horizontal distance between CUAVi and EUi is denoted as $D_{i i}$ and that between SUAV and EUi is denoted as $R_{s i}$. Using the triangle Pythagorean theorem, we can get $d_{i i}$ and $r_{s i}$. 


$$
\begin{aligned}
& d_{i i}=\sqrt{H_{0}^{2}+D_{i i}^{2}}, \\
& r_{s i}=\sqrt{H_{0}^{2}+R_{s i}^{2}} .
\end{aligned}
$$

\section{CGPC Algorithm}

3.1. CGPC Game Elements. In the noncooperative game, each participant will choose selfishness to maximise their benefits, thereby causing interference to the other participants and reducing the benefits to other users. Meanwhile, a cooperative game is meant to maximise the interests of the collective and is a competition model in which the participants seek to maximise their interests. Therefore, a cooperative game will bring more benefits to the system.

The three basic elements of the cooperative game are the participants, strategy set, and utility function. Participants in the game are represented as $I$ : the set of CUAVs participating in the game, where $I=\{1,2, \ldots, N\}$. The strategy set is represented as $\Omega_{i}$ : decision of each participant can be expressed as $\left\{p_{1}, p_{2}, \ldots, p_{i}, \ldots, p_{n}\right\}, 0 \leq p_{i} \leq p_{\mathrm{CUAV}}^{\max }$ (here, $p_{\mathrm{CUAV}}^{\max }$ represents the maximum allowable transmitted power of the CUAV), and each decision is independent of the others. The utility function is given as $U_{i}$, which represents the preference of EUi for a certain strategy.

3.2. Utility Function. In the traditional cooperative game algorithm, the utility function only considers the user SINR. In this study, we consider not only the SINR of users but also the influence of SUAV on EUi. A new utility function is divided into two types according to whether the SINR of users is greater than the threshold SINR:

$$
U_{i}\left(p_{i}, \gamma_{i}\right)= \begin{cases}\prod_{i=1}^{m} g_{s i} \frac{\gamma_{i}-\gamma_{\mathrm{EU}}^{\min }}{\gamma_{i}}, & \gamma_{i} \geq \gamma_{\mathrm{EU}}^{\min }, \\ 0, & \gamma_{i}<\gamma_{\mathrm{EU}}^{\min }\end{cases}
$$

In the first case, when user $\gamma_{i} \geq \gamma_{\mathrm{EU}}^{\min }, U_{i}\left(p_{i}, \gamma_{i}\right)$ $=\prod_{i=1}^{m} g_{s i}\left(\gamma_{i}-\gamma_{\mathrm{EU}}^{\min } / \gamma_{i}\right)$, where $m$ is defined as the number of $\gamma_{i} \geq \gamma_{\mathrm{EU}}^{\min }$ in all UAVs, and $\gamma_{\mathrm{EU}}^{\min }$ is defined as the minimum SINR required for EU communication. $\gamma_{i}-\gamma_{\mathrm{EU}}^{\min }$ guaranteed communication QoS of EU. Only when $\gamma_{i} \geq \gamma_{\mathrm{EU}}^{\min }$ can the utility function be guaranteed to be positive. $\left(\gamma_{i}-\gamma_{\mathrm{EU}}^{\min } / \gamma_{i}\right)$ can make the power change of user EUi smoother and reduce the times of games. It can be observed from (3) that the utility function EUi is related to $g_{s i}$ and $\gamma_{\mathrm{EU}}^{\min }$. The utility function considers the SINR of EUi and the channel gain of the SUAV and EUi. The physical meaning of utility function is to ensure the maximisation of $\left(\gamma_{i}-\gamma_{\mathrm{EU}}^{\min } / \gamma_{i}\right)$ of all EUi.

In the second case, when user $\gamma_{i}<\gamma_{\mathrm{EU}}^{\min }, \gamma_{i}$ cannot be guaranteed the normal communication requirements of $\mathrm{EUi}$, so the utility function of EUi is defined as 0 .

The objective function of power control based on the cooperative game in CGPC can be expressed as follows:

$$
\left\{\begin{array}{l}
\max U\left(p_{i}, \gamma_{i}\right)=g_{s i} \prod_{i=1}^{m} \frac{\gamma_{i}-\gamma_{\mathrm{EU}}^{\min }}{\gamma_{i}}, \\
\text { s.t }\left\{\begin{array}{l}
0 \leq p_{i} \leq p_{\mathrm{CUAV}}^{\max }, \\
\sum_{j=1, j \neq i}^{m} h_{j i} p_{i} \leq T, \\
\gamma_{i} \geq \gamma_{\mathrm{EU}}^{\min } .
\end{array}\right.
\end{array}\right.
$$

Here, $T$ is the threshold value for which EUi can withstand interference from the UAVG. In theory, we set $\gamma_{\mathrm{EU}}^{\min }$ and determine the interference threshold $T$ according to the channel gain and transmit power from EUi to CUAV. $\left(p_{i} h_{i i} / T\right) \geq \gamma_{\mathrm{EU}}^{\min }$, if and only if $\gamma_{i}=\gamma_{\mathrm{EU}}^{\min }$; we can calculate the threshold $T=\left(p_{i} h_{i i} / \gamma_{\mathrm{EU}}^{\min }\right)$. The physical meaning of power control in CGPC indicates that the utility function of the EU reaches the maximum when the interference from CUAV to SU does not exceed the maximum interference threshold that the SU can bear.

3.3. Nash Solution. Equation (4) can be further modified by considering the logarithm of the objective function of (4) and using the properties of logarithmic functions, $\ln \prod_{i=1}^{N} \gamma_{i}=\sum_{i=1}^{N} \ln \gamma_{i}$, as follows:

$$
\left\{\begin{array}{l}
\max U\left(p_{i}, \gamma_{i}\right)=g_{s i} \prod_{i=1}^{m} \ln \left(\frac{\gamma_{i}-\gamma_{\mathrm{EU}}^{\min }}{\gamma_{i}}\right), \\
\text { s.t }\left\{\begin{array}{l}
0 \leq p_{i} \leq p_{\mathrm{CUAV}}^{\max }, \\
\sum_{j=1, j \neq i}^{m} h_{j i} p_{i} \leq T, \\
\gamma_{i} \geq \gamma_{\mathrm{EU}}^{\min } .
\end{array}\right.
\end{array}\right.
$$

Therefore, the problem of $U_{i}\left(p_{i}, \gamma_{i}\right)$ maximisation is now transformed into the problem of $u_{i}\left(p_{i}, \gamma_{i}\right)$ maximisation, and $u_{i}=\ln U_{i}$.

Theorem 1. Model equivalence theorem. Suppose that the decision set for the utility function set has a one-to-one mapping relationship that satisfies the concave function characteristics; that is, $u_{i}=\ln U_{i}$ satisfies the characteristics of the concave function. Thus, it can be concluded that the models in (4) and (5) are equivalent, and the same solution is obtained.

Equation (5) is an optimisation problem with multiple constraints, and the Lagrange factor $\lambda, \mu_{i}, \eta_{i}, \varepsilon_{i}$ is introduced to construct a Lagrange function. Among them, Lagrange factor is the limiting factor set when solving Lagrange function. Through Lagrange factor, the objective function 
and constraint conditions are connected together to construct a new Lagrange function.

Therefore, Lagrange function can be expressed as follows:

$$
\begin{aligned}
L\left(p_{i}, \gamma_{i}\right)= & g_{s i} \sum_{i=1}^{m} \ln \left(\frac{\gamma_{i}-\gamma_{\mathrm{EU}}^{\mathrm{min}}}{\gamma_{i}}\right)-\lambda\left(\sum_{j=1, j \neq i}^{m} h_{j i} p_{i}-T\right) \\
& -\mu_{i}\left(\gamma_{i}-\gamma_{\mathrm{EU}}^{\min }\right)-\eta_{i}\left(p_{\mathrm{CUAV}}^{\max }-p_{i}\right)-\varepsilon_{i}\left(0-p_{i}\right) .
\end{aligned}
$$

According to (6), the partial derivative of the Lagrange function $p_{i}$ is calculated, and the optimal solution is obtained by setting $\left(\partial L\left(p_{i}, \gamma_{i}\right) / \partial p_{i}\right)=0$; so we can get $p_{i}$ as follows:

$$
p_{i}=\frac{\gamma_{\mathrm{EU}}^{\mathrm{min}}}{\gamma_{i}} p_{i}+\frac{g_{s i}}{\mu_{i}\left(\partial \gamma_{i} / \partial p_{i}\right)-\lambda\left(\sum_{j=1, j \neq i}^{m} h_{j i} p_{i}-T\right)-\left(\eta_{i}+\varepsilon_{i}\right)} .
$$

According to the SINR definition, $\left(\partial \gamma_{i} / \partial p_{i}\right)=\left(\gamma_{i} / p_{i}\right)$ holds. The fixed-point iterative method is used for iteration; after $k$ iterations, the transmitted power of CUAVi is as follows:

$$
p_{i}^{(k+1)}=\frac{\gamma_{\mathrm{EU}}^{\min }}{\gamma_{i}} p_{i}^{(k)}+\frac{g_{s i}}{\mu_{i}\left(\gamma_{i} / p_{i}^{(k)}\right)-\lambda\left(\sum_{j=1, j \neq i}^{m} h_{j i} p_{i}-T\right)-\left(\eta_{i}+\varepsilon_{i}\right)} .
$$

According to (8), the first term $\left(\gamma_{\mathrm{EU}}^{\min } / \gamma_{i}\right) p_{i}^{(k)}$ on the right is consistent with the traditional SINR balancing algorithm [12], and it is expressed that $p_{i}^{(k+1)}$ will gradually stabilise when $\gamma_{i}$ approaches $\gamma_{\mathrm{EU}}^{\min }$. The second term $\left(g_{s i} / \mu_{i}\left(\gamma_{i} / p_{i}^{(k)}\right)-\lambda\right.$ $\left.\left(\sum_{j=1, j \neq i}^{m} h_{j i} p_{i}-T\right)-\left(\eta_{i}+\varepsilon_{i}\right)\right)$ is a fine-tuning term comprising the Lagrangian factors, channel gain, and current SINR. This proves the correctness of the algorithm. It not only ensures that it is similar to the traditional SINR balancing algorithm but also makes fine-tuning on the basis of the traditional SINR balancing algorithm. The algorithm in this paper is based on UAV transmit power polling, not on time.

In this paper, the throughput of the system is characterised by the sum of the average reachable rates of all users, where the average reachable rate of each user can be expressed as

$$
R_{i}=B \log _{2}\left(1+\gamma_{i}\right)
$$

where $R_{i}$ is the user reachable rate and the bandwidth is $B=1 \mathrm{~Hz}$. Therefore, the system throughput of each iteration can be expressed as follows:

$$
S_{t}^{(k)}=\sum_{i=1}^{N} R_{i}^{(k)}
$$

where $S_{t}^{(k)}$ is the throughput of the $k$-th iteration. $R_{i}^{(k)}$ is the user reachable rate of user $i$ at the $k$-th iteration.

\section{Performance Evaluation}

In this section, we present verification of the feasibility of the CGPC algorithm through simulations and calibrations for the theoretical analysis results and simulation results.

4.1. Simulation Configuration and Parameters. According to [11-13], the height of the UAVG is selected as $H_{0}=80 \mathrm{~m}$, and the horizontal projection coverage area of the UAVG is selected in a circular area of radius $300 \mathrm{~m}$, where the horizontal distance from the SU to SUAV is set as $[0,100 \mathrm{~m}]$, and the horizontal distance from EU to SUAV is set as $(100,300]$. Suppose that there is one SUAV and four CUAVs, namely, CUAV1, CUAV2, CUAV3, and CUAV4, in this circular area. In the circular region, there is one SU and four EU, namely, EU1, EU2, EU3, and EU4. The distance relationship between the EUs and SUAV is expressed as $R_{s 1} \leq R_{s 2} \leq R_{s 3} \leq R_{s 4}$.

Suppose that the transmitted power of the SUAV is $30 \mathrm{dBm}$, and the initial transmitted power of the CUAV is $20 \mathrm{dBm}$. Assuming noise threshold $T=1 \times 10^{-10} \mathrm{~W}$, the Lagrange factors are obtained as $\lambda=1 \times 10^{16}$ and $\mu_{i}=\eta_{i}=$ $\varepsilon_{i}=10$ [11-13].

4.2. Simulation Results and Analysis. Figure 2 illustrates the curves for the CUAV transmitted power changing with the number of iterations. The abscissa in the figure is the number of iterations, and the ordinate is the transmitted power from the CUAV. It can be observed from the figure that the initial transmitting power of CUAVs is $0.1 \mathrm{~W}$. With the increase of iteration times, the transmitting power of CUAV decreases gradually and reaches a stable state after many games. The attenuation rate of CUAV1 is the fastest and that of CUAV4 is the slowest. This is due to the different distances between EU and SU. The distance between EU1 and SU is relatively close, and the downlink communication power of CUAV1 and EU1 has a greater impact on SU. The power attenuation of CUAV1 is relatively large. The distance between EU4 and SU is relatively long, and the influence of CUAV 4 and EU4 downlink communication power on SU is relatively small. Therefore, the power attenuation of CUAV 4 is relatively small. This is consistent with the result of formula (3).

Figure 3 presents a comparison of the CUAV transmitted power for different algorithms. The abscissa is the distance between the EU and SUAV, and the ordinate is the transmitted power from the CUAV. The curves in the figure represent the results of the K-G algorithm [12] and CGPC algorithm. It can be observed that the transmitted power of the CUAV increases with the distance between the EU and SUAV. When the distance between EU and CUAV is close, 


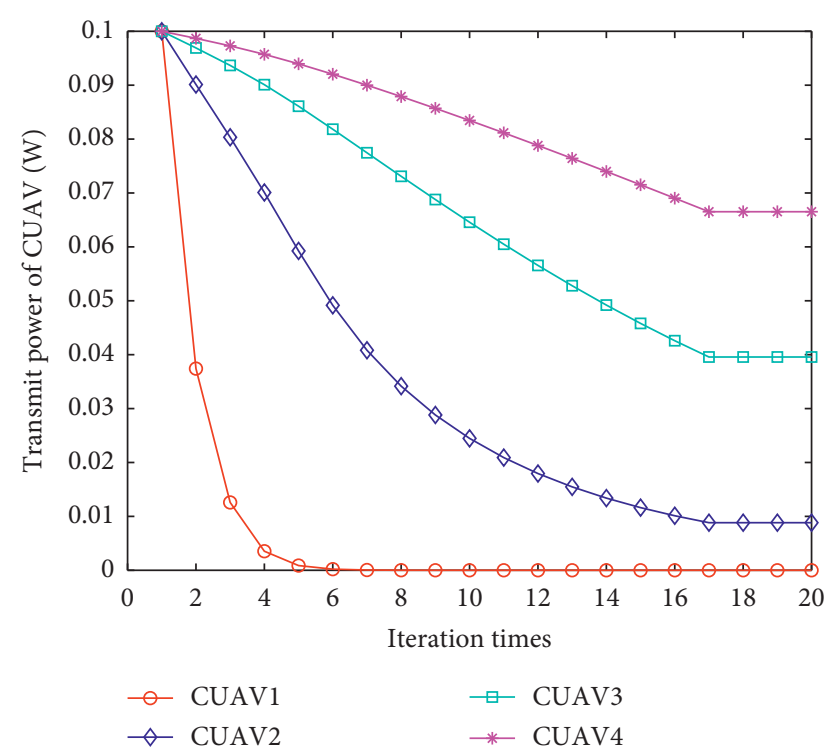

FIgURE 2: Transmitted power from CUAV for number of iterations.

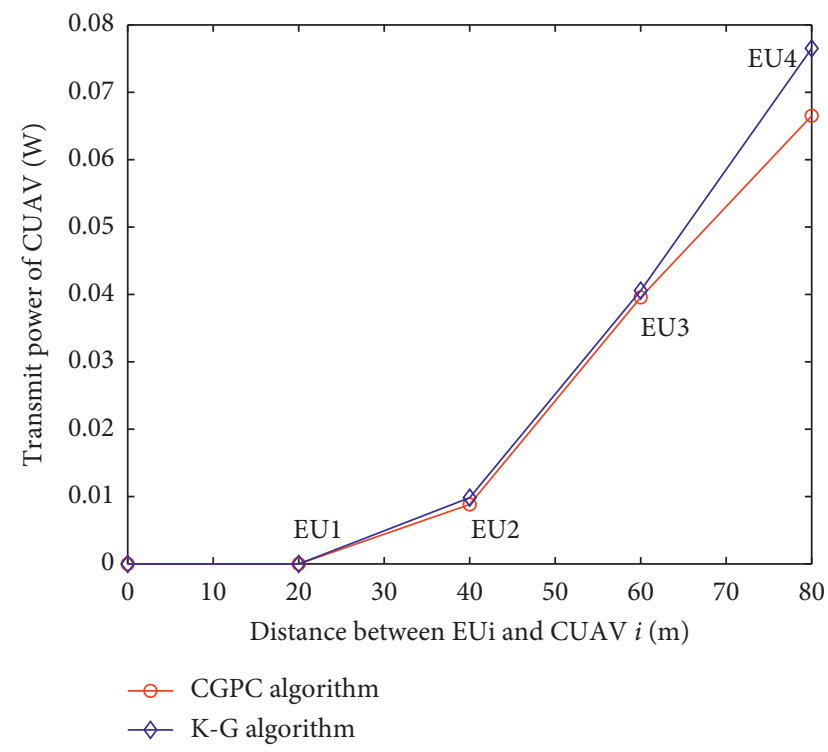

FIgURE 3: Comparison of cooperative UAV transmitted power under different algorithms.

the effect of the proposed algorithm is equivalent to that of the traditional K-G algorithm. However, after 60 meters, it obviously reflects the advantages of this algorithm, which can effectively reduce the transmission power of CUAV. This is because the design of utility function considers not only the user's QoS but also the influence of different distance EU on CUAV. The farther the distance between EU and CUAV, the more obvious the advantages of this algorithm. Comparing these two algorithms under the same conditions, the transmitted power of the CUAV based on the CGPC algorithm is observed to be lower than that of the K-G algorithm, which reduces the cofrequency interference.

Figure 4 demonstrates the curves for the system average throughput according to the number of CUAVs for different

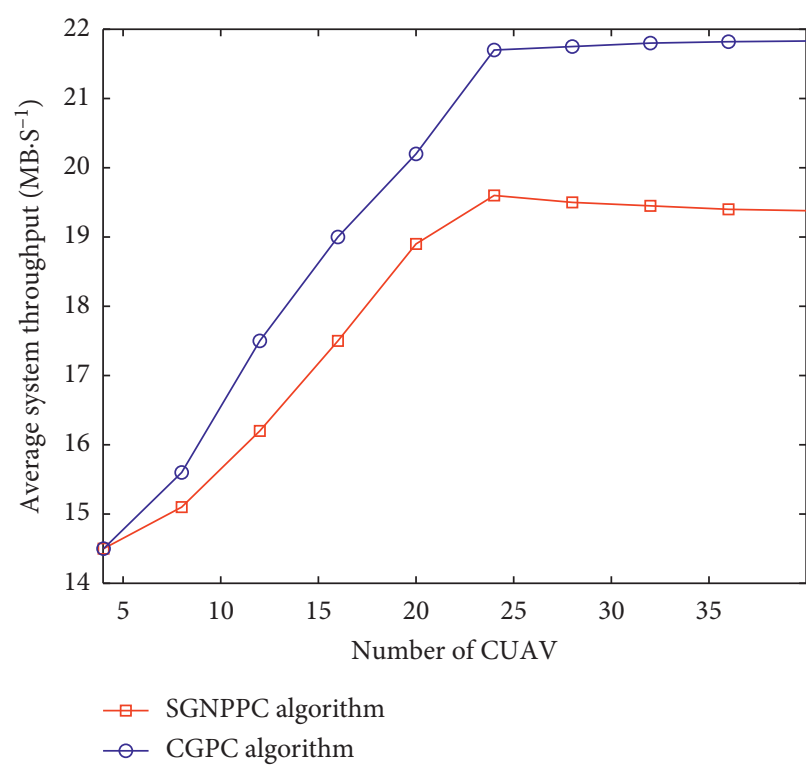

FIgURE 4: Transmitted power for the system average throughput CUAV for different algorithms.

algorithms; the abscissa is the number of CUAVs, and the ordinate is the average throughput of the system. The curves represent results for the Stackelberg Game based Nonunified Pricing Power Control (SGNPPC) algorithm [13] and CGPC algorithm for the average system throughput. It can be observed that the average throughput of the two systems increases as the number of CUAVs increases. When the number of CUAVs reaches 24, the average throughput of the system is close to saturation. Thereafter, the system throughput of the SGNPPC algorithm gradually decreases with increase in the number of CUAVs; this is because more interference is inserted with the increase in the number of CUAVs; conversely, the throughput of the CGPC algorithm increases with the increase in the number of CUAVs, but the rate of increase is reduced. This deceleration can be attributed to the adoption of cooperative games to reduce the interference between the CUAVs. Compared with the SGNPPC algorithm, the average throughput of the CGPC algorithm increased by $10.32 \%$.

\section{Conclusion}

In this study, a CGPC algorithm is proposed in the scenario of the UUAVG. The algorithm considers not only the SINR of the user but also the influence of the different distances between the EU and SUAV on the EU. The complex Nash theorem utility function is transformed into an easily solved optimisation problem using the principle of model equivalence; further, the Lagrange function is constructed, and the optimal transmitted power is obtained iteratively. The convergence of the algorithm is proven by simulation, and the average throughput of the system is improved. In the future work, we will consider the joint optimisation of distributed power control and user rate control, as well as the trajectory design and layout design of UAV. 


\section{Data Availability}

No data were used to support this study.

\section{Conflicts of Interest}

The authors declare that there are no conflicts of interest regarding the publication of this paper.

\section{Acknowledgments}

This work was supported by the subproject of National Key Research and Development Plan in 2020 (no. 2020YFC1511704), the National Natural Science Foundation of China (Grant no. 61971048), Beijing Science and Technology Project (Grant no. Z191100001419012), and scientific research level improvement project to promote the colleges connotation development of Beijing Information Science \& Technology University in 2020 (no. 2020KYNH212).

\section{References}

[1] H. Menouar, I. Guvenc, K. Akkaya, A. S. Uluagac, A. Kadri, and A. Tuncer, "UAV-enabled intelligent transportation systems for the smart city: applications and challenges," IEEE Communications Magazine, vol. 55, no. 3, pp. 22-28, 2017.

[2] W. Huang, J. Peng, and H. Zhang, "User-centric intelligent UAV swarm networks: performance analysis and design insight," IEEE Access, vol. 7, pp. 181469-181478, 2019.

[3] S. Chen, F. Qin, B. Hu, X. Li, and Z. Chen, "User-centric ultradense networks for 5G: challenges, methodologies, and directions," IEEE Wireless Communications, vol. 23, no. 2, pp. 78-85, 2016.

[4] R. Tang, J. Zhao, H. Qu et al., "User-centric joint admission control and resource allocation for 5G D2D extreme mobile broadband: a sequential convex programming approach," IEEE Communications Letters, vol. 7, 2017.

[5] P. Zhang and Y. Zhang, "Research on power control algorithm of two-layer Stackelberg game in UUDN," Computer Engineering, vol. 46, no. 9, pp. 186-192, 2020, in Chinese.

[6] H. Zhang and W. Huang, "Tractable mobility model for multiconnectivity in 5G user-centric ultra-dense networks," IEEE Access, vol. 6, pp. 43100-43112, 2018.

[7] S. Bhattacharya and T. Basart, "Game-theoretic analysis of an aerial jamming attack on a UAV communication network," in Proceeding of the American Control Conference, pp. 818-823, IEEE, Baltimore, MD, USA, July 2010.

[8] Q. Li, "Non-cooperative game power control in swarm UAV networks," Telecommunication Engineering, vol. 7, 2019, in Chinese.

[9] W. Mei, Q. Wu, and R. Zhang, "Cellular-connected UAV: uplink association, power control and interference coordination," in Proceedings of the IEEE Global Communications Conference, Abu Dhabi, UAE, December 2018.

[10] Y. Shi, M. Peng, and X. Cao, "A game theory approach for joint access selection and resource allocation in UAV assisted IoT communication networks," IEEE Internet of Things Journal, vol. 99, 2018.

[11] L. Li, Q. Cheng, K. Xue, C. Yang, and Z. Han, "Downlink transmit power control in ultra-dense UAV network based on mean field game and deep reinforcement learning," IEEE Transactions on Vehicular Technology, vol. 69, no. 12, pp. 15594-15605, 2020.
[12] Z. R. Gajic and S. Koskie, "Newton iteration acceleration of the Nash game algorithm for power control in $3 \mathrm{G}$ wireless CDMA networks," in Proceedings, ITCOM 2003, Conference on Performance and Control of Next Generation of Communication Networks, no. 5, pp. 115-121, Orlando, FL, USA, August 2003.

[13] C. M. XU and J. WU, "Non- unified pricing power control based on the Stackelberg game in the ultra-dense network," Journal of Computer Applications, vol. 38, no. 08, pp. 23232329, 2018, in Chinese. 